\title{
Hyperbolicity and chordality of a graph
}

\author{
Yaokun $\mathrm{Wu}^{*}$ and Chengpeng Zhang \\ Department of Mathematics, Shanghai Jiao Tong University \\ 800 Dongchuan Road, Shanghai, 200240, China
}

Submitted: Oct 27, 2009; Accepted: Feb 7, 2011; Published: Feb 21, 2011

Mathematics Subject Classifications: 05C05, 05C12, 05C35, 05C62, 05C75

\begin{abstract}
Let $G$ be a connected graph with the usual shortest-path metric $d$. The graph $G$ is $\delta$-hyperbolic provided for any vertices $x, y, u, v$ in it, the two larger of the three sums $d(u, v)+d(x, y), d(u, x)+d(v, y)$ and $d(u, y)+d(v, x)$ differ by at most $2 \delta$. The graph $G$ is $k$-chordal provided it has no induced cycle of length greater than $k$. Brinkmann, Koolen and Moulton find that every 3-chordal graph is 1-hyperbolic and that graph is not $\frac{1}{2}$-hyperbolic if and only if it contains one of two special graphs as an isometric subgraph. For every $k \geq 4$, we show that a $k$-chordal graph must be $\frac{\left\lfloor\frac{k}{2}\right\rfloor}{2}$-hyperbolic and there does exist a $k$-chordal graph which is not $\frac{\left\lfloor\frac{k-2}{2}\right\rfloor}{2}$-hyperbolic. Moreover, we prove that a 5 -chordal graph is $\frac{1}{2}$-hyperbolic if and only if it does not contain any of a list of five special graphs as an isometric subgraph.
\end{abstract}

Keywords: isometric subgraph; metric; tree-likeness.

\section{Introduction}

\section{$1.1 \quad$ Tree-likeness}

Trees are graphs with some very distinctive and fundamental properties and it is legitimate to ask to what degree those properties can be transferred to more general structures that are tree-like in some sense [28, p. 253]. Roughly speaking, tree-likeness stands for something related to low dimensionality, low complexity, efficient information deduction (from local to global), information-lossless decomposition (from global into simple pieces) and nice shape for efficient implementation of divide-and-conquer strategy. For the very basic interconnection structures like a graph or a hypergraph, tree-likeness is naturally reflected by the strength of interconnection, namely its connectivity/homotopy type or cyclicity/acyclicity, or just the degree of deviation from some characterizing conditions of a tree/hypertree and its various associated structures and generalizations. In vast

*Corresponding author. Email: ykwu@sjtu.edu.cn. 
applications, one finds that the borderline between tractable and intractable cases may be the tree-like degree of the structure to be dealt with [18]. A support to this from the fixed-parameter complexity point of view is the observation that on various tree-structures we can design very good algorithms for many purposes and these algorithms can somehow be lifted to tree-like structures $[4,31,32,62]$. It is thus very useful to get information on approximating general structures by tractable structures, namely tree-like structures. On the other hand, one not only finds it natural that tree-like structures appear extensively in many fields, say biology [38], structured programs [75] and database theory [40], as graphical representations of various types of hierarchical relationships, but also notice surprisingly that many practical structures we encounter are just tree-like, say the internet $[1,60,73]$ and chemical compounds [80]. This prompts in many areas the very active study of tree-like structures. Especially, lots of ways to define/measure a tree-like structure have been proposed in the literature from many different considerations, just to name a few, say asymptotic connectivity [5], boxicity [69], combinatorial dimension [34, 38], coverwidth [19], cycle rank [18, 65], Domino treewidth [9], doubling dimension [50], $\epsilon-$ three-points condition [29], $\epsilon$-four-points condition [1], hypertree-width [48], Kelly-width [54], linkage (degeneracy) [26, 58, 66], McKee-Scheinerman chordality [67], persistence [31], s-elimination dimension [26], sparsity order [63], spread-cut-width [24], tree-degree

[17], tree-length [30, 77], tree-partition-width [79], tree-width [70, 71], various degrees of acyclicity/cyclicity [39, 40], and many other width parameters $[32,52]$. It is clear that many relationships among these concepts should be expected as they are all formulated in different ways to represent different aspects of our vague but intuitive idea of tree-likeness. An attempt to clarify these relationships may help to bridge the study in different fields focusing on different tree-likeness measures and help to improve our understanding of the universal tree-like world. As a small step in pursuing further understanding of treelikeness, we take up in this paper the modest task of comparing two parameters of treelikeness, namely (Gromov) hyperbolicity and chordality of a graph. We discuss these two parameters separately in the next two subsections. We then close this section with a summary of known relationship between them and an outlook for some further research.

\subsection{Hyperbolicity}

We only consider simple, unweighted, connected, but not necessarily finite graphs. Any graph $G$ together with the usual shortest-path metric on it, $d_{G}: V(G) \times V(G) \mapsto$ $\{0,1,2, \ldots\}$, gives rise to a metric space. We often suppress the subscript and write $d(x, y)$ instead of $d_{G}(x, y)$ when the graph is known by context. Moreover, we may use the shorthand $x y$ for $d(x, y)$ to further simplify the notation. Note that a pair of vertices $x$ and $y$ form an edge if and only if $x y=1$. For $S, T \subseteq V(G)$, we write $d(S, T)$ for $\min _{x \in S, y \in T} d(x, y)$. We often omit the brackets and adopt the convention that $x$ stands for the singleton set $\{x\}$ when no confusion can be caused. A subgraph $H$ of a graph $G$ is isometric if for any $u, v \in V(H)$ it holds $d_{H}(u, v)=d_{G}(u, v)$.

For any vertices $x, y, u, v$ of a graph $G$, put $\delta_{G}(x, y, u, v)$, which we often abbreviate to $\delta(x, y, u, v)$, to be the difference between the largest and the second largest of the following 
three terms:

$$
\frac{u v+x y}{2}, \frac{u x+v y}{2} \text {, and } \frac{u y+v x}{2} .
$$

Clearly, $\delta(x, y, u, v)=0$ if $x, y, u, v$ are not four different vertices. A graph $G$, viewed as a metric space as mentioned above, is $\delta$-hyperbolic (or tree-like with defect at most $\delta$ ) provided for any vertices $x, y, u, v$ in $G$ it holds $\delta(x, y, u, v) \leq \delta$ and the (Gromov) hyperbolicity of $G$, denoted $\delta^{*}(G)$, is the minimum half integer $\delta$ such that $G$ is $\delta$-hyperbolic $[11,13,21,22,27,49]$. Note that it may happen $\delta^{*}(G)=\infty$. But for a finite graph $G$, $\delta^{*}(G)$ is clearly finite and polynomial time computable. A graph $G$ is minimally $\delta$ hyperbolic if $\delta=\delta^{*}(G)$ and any isometric proper subgraph of $G$ is $\left(\delta-\frac{1}{2}\right)$-hyperbolic. Similarly, a graph $G$ is minimally non- $\delta$-hyperbolic if $\delta<\delta^{*}(G)$ and any isometric proper subgraph of $G$ is $\delta$-hyperbolic.

Note that in some earlier literature the concept of Gromov hyperbolicity is used a little bit different from what we adopt here; what we call $\delta$-hyperbolic here is called $2 \delta$ hyperbolic in $[1,6,7,14,23,35,38,44,61,68]$ and hence the hyperbolicity of a graph is always an integer according to their definition. We also refer to $[2,11,13,78]$ for some equivalent and very accessible definitions of Gromov hyperbolicity which involve some other comparable parameters.

The concept of hyperbolicity comes from the work of Gromov in geometric group theory which encapsulates many of the global features of the geometry of complete, simply connected manifolds of negative curvature [13, p. 398]. This concept not only turns out to be strikingly useful in coarse geometry but also becomes more and more important in many applied fields like networking and phylogenetics $[20,21,22,23,33,34,35,36$, $38,44,56,57,60,73]$. The hyperbolicity of a graph is a way to measure the additive distortion with which every four-points sub-metric of the given graph metric embeds into a tree metric [1]. Indeed, it is not hard to check that the hyperbolicity of a tree is zero - the corresponding condition for this is known as the four-point condition (4PC) and is a characterization of general tree-like metric spaces $[34,38,55]$. Moreover, the fact that hyperbolicity is a tree-likeness parameter is reflected in the easy fact that the hyperbolicity of a graph is the maximum hyperbolicity of its 2-connected components - This observation implies the classical result that 0-hyperbolic graphs are exactly block graphs, namely those graphs in which every 2-connected subgraph is complete, which are also known to be those diamond-free chordal graphs $[8,37,53]$. More results on bounding hyperbolicity of graphs and characterizing low hyperbolicity graphs can be found in $[6,7,14,20,21,30,61]$.

For any vertex $u \in V(G)$, the Gromov product, also known as the overlap function, of any two vertices $x$ and $y$ of $G$ with respect to $u$ is equal to $\frac{1}{2}(x u+y u-x y)$ and is denoted by $(x \cdot y)_{u}[13$, p. 410]. As an important context in phylogenetics [35, 36, 42], for any real number $\rho$, the Farris transform based at $u$, denoted $D_{\rho, u}$, is the transformation which sends $d_{G}$ to the map

$$
D_{\rho, u}\left(d_{G}\right): V(G) \times V(G) \rightarrow \mathbb{R}:(x, y) \mapsto \rho-(x \cdot y)_{u}
$$

We say that $G$ is $\delta$-hyperbolic with respect to $u \in V(G)$ if the following inequality

$$
(x \cdot y)_{u} \geq \min \left((x \cdot v)_{u},(y \cdot v)_{u}\right)-\delta
$$


holds for any vertices $x, y, v$ of $G$. The inequality (1) can be rewritten as

$$
x y+u v \leq \max (x u+y v, x v+y u)+2 \delta
$$

and so we see that $G$ is $\delta$-hyperbolic if and only if $G$ is $\delta$-hyperbolic with respect to every vertex of $G$. By a simple but nice argument, Gromov shows that $G$ is $2 \delta$-hyperbolic provided it is $\delta$-hyperbolic with respect to any given vertex [2, Proposition 2.2] [49, 1.1B].

\subsection{Chordality}

Let $G$ be a graph. A walk of length $n$ in $G$ is a sequence of vertices $x_{0}, x_{1}, x_{2}, \ldots, x_{n}$ such that $x_{i-1} x_{i}=1$ for $i=1, \ldots, n$. If these $n+1$ vertices are pairwise different, we call the sequence a path of length $n$. A cycle of length $n$, or simply an $n$-cycle, in $G$ is a cyclic sequence of $n$ different vertices $x_{1}, \ldots, x_{n} \in V(G)$ such that $x_{i} x_{j}=1$ whenever $j=i+1$ $(\bmod n)$; we will reserve the notation $\left[x_{1} x_{2} \cdots x_{n}\right]$ for this cycle. A chord of a cycle is an edge joining nonconsecutive vertices on the cycle. A cycle without chord is called an induced cycle, or a chordless cycle. For any $n \geq 3$, the $n$-cycle graph is the graph with $n$ vertices which has a chordless $n$-cycle and we denote this graph by $C_{n}$.

We say that a graph is $k$-chordal if it does not contain any induced $n$-cycle for $n>k$. Clearly, trees are nothing but 2-chordal graphs. A 3-chordal graph is usually termed as a chordal graph and a 4-chordal graph is often called a hole-free graph. The class of $k$-chordal graphs is also discussed under the name $k$-bounded-hole graphs [45]. The chordality of a graph $G$ is the smallest integer $k \geq 2$ such that $G$ is $k$-chordal [10]. Following [10], we use the notation $\mathbb{1} \mathbb{C}(G)$ for this parameter as it is merely the length of the longest chordless cycle in $G$ when $G$ is not a tree. Note that our use of the concept of chordality is basically the same as that used in $[15,16]$ but is very different from the usage of this term in [67].

The recognition of $k$-chordal graphs is coNP-complete for $k=\Theta\left(n^{\epsilon}\right)$ for any constant $\epsilon>0$ [76]. Especially, to determine the chordality of the hypercube is attracting much attention under the name of the snake-in-the-box problem due to its connection with some error-checking codes problem [59]. Nevertheless, just like many other tree-likeness parameters, quite a few natural graph classes are known to have small chordality [12]; also see Section 5 .

\section{$1.4 \quad$ Hyperbolicity versus chordality}

Firstly, we point out that a graph with low hyperbolicity may have large chordality. Indeed, take any graph $G$ and form the new graph $G^{\prime}$ by adding an additional vertex and connecting this new vertex with every vertex of $G$. It is obvious that we have $\delta^{*}\left(G^{\prime}\right) \leq 1$ and $\mathbb{1} \mathbb{C}\left(G^{\prime}\right)=\mathbb{1} \mathbb{C}(G)$ as long as $G$ is not a tree. Moreover, it is equally easy to see that $G^{\prime}$ is even $\frac{1}{2}$-hyperbolic if $G$ does not have any induced 4-cycle [61, p. 695]. Surely, this example does not preclude the possibility that for many important graph classes we can bound their chordality in terms of their hyperbolicity. 


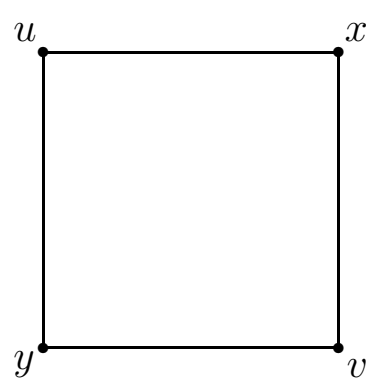

$C_{4}$

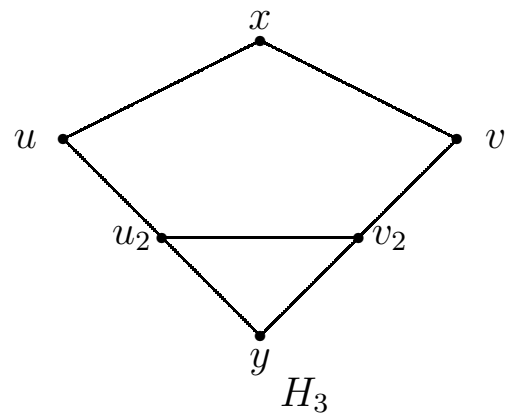

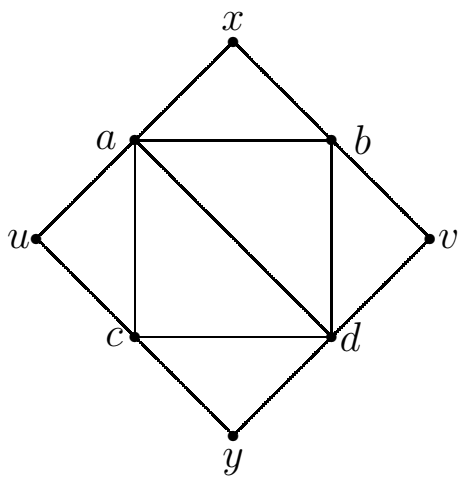

$H_{1}$

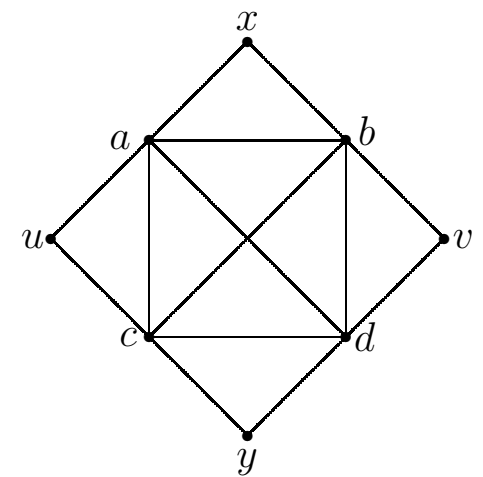

$\mathrm{H}_{2}$

Figure 1: Five 5-chordal graphs with hyperbolicity 1. 
Let $C_{4}, H_{1}, H_{2}, H_{3}$ and $H_{4}$ be the graphs displayed in Fig. 1. It is simple to check that

$$
\left\{\begin{array}{l}
\mathbb{I}_{\mathbb{C}}\left(H_{1}\right)=3, \mathbb{I}_{\mathbb{C}}\left(H_{2}\right)=3, \mathbb{1}_{\mathbb{C}}\left(C_{4}\right)=4, \mathbb{I}_{\mathbb{C}}\left(H_{3}\right)=5, \mathbb{l}_{\mathbb{C}}\left(H_{4}\right)=5 ; \\
\delta^{*}\left(H_{1}\right)=\delta^{*}\left(H_{2}\right)=\delta^{*}\left(C_{4}\right)=\delta^{*}\left(H_{3}\right)=\delta^{*}\left(H_{4}\right)=1 .
\end{array}\right.
$$

Brinkmann, Koolen and Moulton obtain the following interesting result.

Theorem 1 [14, Theorem 1.1] Every chordal graph is 1-hyperbolic and it has hyperbolicity one if and only if it contains either $\mathrm{H}_{1}$ or $\mathrm{H}_{2}$ as an isometric subgraph.

Now, we come to the general observation that $k$-chordal graphs have bounded hyperbolicity for any fixed $k$, generalizing the corresponding fact reported in Theorem 1 for $k=3$. Note that a chordal graph is certainly 4 -chordal and $\frac{\left\lfloor\frac{k}{2}\right\rfloor}{2}$ is just 1 for $k=4$.

Theorem 2 For each $k \geq 4$, all $k$-chordal graphs are $\frac{\left\lfloor\frac{k}{2}\right\rfloor}{2}$-hyperbolic.

For any given integer $k \geq 4$, we can find graphs $G$ of chordality $k$ such that the equality

$$
\delta^{*}(G)=\frac{\left\lfloor\frac{\mathbb{C}(G)}{2}\right\rfloor}{2}
$$

holds; see Section 4. In this sense, the inequality obtained in Theorem 2 is tight. Surely, the logical next step would be to characterize all those extremal graphs $G$ satisfying Eq. (3). However, there seems to be still a long haul ahead in this direction. A graph is bridged $[3,64]$ if it does not contain any finite isometric cycles of length at least four. In contrast to Theorem 2, it is interesting to note that the hyperbolicity of bridged graphs can be arbitrarily high $[61$, p. 684$]$.

We know that a graph with small hyperbolicity can be said to be very tree-like. But how do these tree-like graphs look alike? Or, "what is the structure of graphs with relative small hyperbolicity" [14, p. 62]? As mentioned in Section 1.2, the structure of 0 -hyperbolic graphs is well-understood. The next important step forward in this direction is the characterization of all $\frac{1}{2}$-hyperbolic graphs obtained by Bandelt and Chepoi [6]. We refer to [6, Fact 1] for two other characterizations; also see [41, 74].

Let $x, y, u, v$ be four vertices in a graph $G$. These four vertices consist of a slingshot from $x$ to $y$ in $G$ provided $x u=x v=1, u v=2$ and $x u+u y=x v+v y=x y$ (and hence $\delta(x, y, u, v) \geq 1)$ and the length of this slingshot is defined to be $x y$. Let $E_{1}, E_{2}, G_{1}, G_{2}$ be the graphs depicted in Fig. 2. Note that

$$
\left\{\begin{array}{l}
\mathbb{I}_{\mathbb{C}}\left(G_{1}\right)=\mathbb{I}_{\mathbb{C}}\left(G_{2}\right)=6, \mathbb{l}_{\mathbb{C}}\left(E_{1}\right)=7, \mathbb{l}_{\mathbb{C}}\left(E_{2}\right)=8 \\
\delta^{*}\left(G_{1}\right)=\delta^{*}\left(G_{2}\right)=\delta^{*}\left(E_{1}\right)=\delta^{*}\left(E_{2}\right)=1
\end{array}\right.
$$

Theorem 3 [6, p. 325] A graph $G$ is $\frac{1}{2}$-hyperbolic if and only if $G$ contains neither any slingshot nor any isometric n-cycle for any $n>5$, and none of the six graphs $H_{1}, H_{2}, G_{1}, G_{2}, E_{1}, E_{2}$ occurs as an isometric subgraph of $G$.

Starting from Theorem 3, it is only a short step to the next result. 

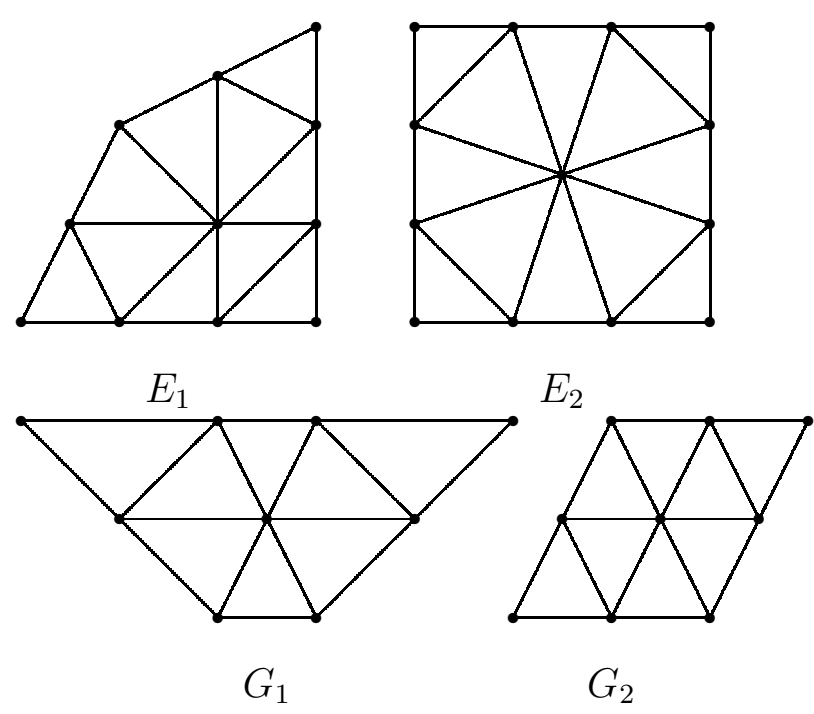

Figure 2: Four bridged graphs with hyperbolicity 1.

Theorem 4 A 5-chordal graph is minimally non- $\frac{1}{2}$-hyperbolic if and only if it is one of $\mathrm{C}_{4}, \mathrm{H}_{1}, \mathrm{H}_{2}, \mathrm{H}_{3}$, or $\mathrm{H}_{4}$.

It is noteworthy that Theorem 2 together with Theorem 4 implies Theorem 1 . Moreover, here is another immediate consequence of Theorems 2 and 4.

Corollary 5 Every 4-chordal graph must be 1-hyperbolic and it has hyperbolicity one if and only if it contains one of $\mathrm{C}_{4}, \mathrm{H}_{1}$ and $\mathrm{H}_{2}$ as an isometric subgraph.

Let $\mathscr{S}_{k}$ stand for the set of all $k$-chordal minimally non- $\frac{1}{2}$-hyperbolic graphs and $\mathscr{S}_{k}^{\prime}$ the set of all $k$-chordal minimally 1-hyperbolic graphs. It is trivially true that $\mathscr{S}_{k}^{\prime} \subseteq \mathscr{S}_{k}$. Notice that Theorem 2 and Theorem 4 assert that $\mathscr{S}_{5}^{\prime}=\mathscr{S}_{5}=\left\{C_{4}, H_{1}, H_{2}, H_{3}, H_{4}\right\}$. We have found that $\mathscr{S}_{6}$ contains quite many elements. In general, it seems to be of interest to investigate the sizes of $\mathscr{S}_{k}$ and $\mathscr{S}_{k}^{\prime}$. When will they become infinite sets? Given a fixed integer $k \geq 4$, another question, which sounds natural due to Theorem 2 , is whether or not there exist infinitely many $k$-chordal graphs which are minimally $\frac{\left\lfloor\frac{k}{2}\right\rfloor}{2}$-hyperbolic.

The plan of the remainder of this paper is as follows. We prove Theorem 4 in Section 2. Then, we deduce Theorem 2 in Section 3 and give examples in Section 4 to show the sharpness of Theorem 2. The last section, Section 5, is devoted to an examination of various low chordality graph classes in algorithmic graph theory from the viewpoint of the hyperbolicity parameter.

\section{Proof of Theorem 4}

In the course of our proof, we will frequently make use of the triangle inequality for the shortest-path metric, namely $a b+b c \geq a c$, without any claim. We also observe that for any induced subgraph $H$ of a graph $G, H$ is an isometric subgraph of $G$ if and only if $d_{H}(u, v)=d_{G}(u, v)$ for each pair of vertices $(u, v) \in V(H) \times V(H)$ satisfying $d_{H}(u, v) \geq 3$. 
Lemma 6 Let $G$ be a graph. Let $C_{4}, H_{3}$ and $H_{4}$ be three graphs as displayed in Fig. 1. (i) If $\mathrm{C}_{4}$ is an induced subgraph of $G$, then it is isometric. (ii) If $\mathrm{H}_{3}$ is an induced subgraph of $G$, then it is isometric if and only if $x y=3$. (iii) If $H_{4}$ is an induced subgraph of $G$, then it is isometric if and only if $u v_{3}=v u_{3}=3$ and $x y=4$.

Proof: Claims (i) and (ii) directly come from the simple observation listed before this lemma. What we have to show is the "if" part of (iii). Based on the fact that $d_{G}(x, y)=4$, we can derive from the triangle inequality that $d_{G}\left(x, u_{3}\right)=d_{G}\left(x, v_{3}\right)=$ $d_{G}(y, u)=d_{G}(y, v)=3$. Since $\left\{u, v_{3}\right\},\left\{v, u_{3}\right\},\left\{x, u_{3}\right\},\left\{x, v_{3}\right\},\{y, u\},\{y, v\},\{x, y\}$ are all pairs inside $\left(\begin{array}{c}V\left(H_{4}\right) \\ 2\end{array}\right)$ which are of distance at least 3 apart in $H_{4}$, the result then follows from the above-mentioned observation, as desired.

Lemma 7 Let $G$ be a graph and suppose that the length of a shortest slingshot in $G$ is $\ell \geq 2$. Let $x, y, u, v$ be a slingshot from $x$ to $y$ and let $P_{u}: u_{0}=x, u_{1}=u, u_{2}, \ldots, u_{\ell}=y$ and $P_{v}: v_{0}=x, v_{1}=v, v_{2}, \ldots, v_{\ell}=y$ be two shortest paths connecting $x$ and $y$. Then the subgraph of $G$ induced by $P_{u} \cup P_{v}$ is either the $2 \ell$-cycle $C=\left[u_{0} u_{1} \cdots u_{\ell} v_{\ell-1} \cdots v_{1}\right]$ or the graph obtained from $C$ by adding one additional edge connecting $u_{i}$ and $v_{i}$ for some $1 \leq i \leq \ell-1$. More precisely, the following hold: ( $i)$ For any $i, j \in\{1,2, \ldots, \ell-1\}$, $u_{i} v_{j}>|i-j|$; (ii) there are no $0<i<j<\ell$ such that $u_{i} v_{i}=u_{j} v_{j}=1$.

Proof: To prove (i), we need only consider the case that $i \leq j$. Note that $u_{i} v_{j}=$ $u_{i} v_{j}+x u_{i}-i \geq x v_{j}-i=j-i=|i-j|$. If equality holds, we have two shortest paths between $x$ and $v_{j}$, one being $v_{0}, v_{1}, \ldots, v_{j}$, the other being $u_{0}, u_{1}, \ldots, u_{i}$, followed by any shortest path from $u_{i}$ to $v_{j}$. This means that there is a slingshot from $x$ to $v_{j}$ of length $j<\ell$, contradicting the minimality of $\ell$ and that is it.

Assume that (ii) were not true. Then, making use of (i), we know that $u_{i}, v_{i}, v_{i+1}, \ldots, v_{j}$ and $u_{i}, u_{i+1}, \ldots, u_{j}, v_{j}$ are two shortest paths connecting $u_{i}$ and $v_{j}$. Appealing to (i) again, we can check that $u_{i}, v_{j}, v_{i}, u_{i+1}$ form a slingshot from $u_{i}$ to $v_{j}$ of length $j-i+1 \leq \ell-1$. This is impossible and so we are done.

Proof of Theorem 4: It is straightforward to see that $C_{4}, H_{1}, H_{2}, H_{3}$, and $H_{4}$ are all 5-chordal and minimally 1-hyperbolic. So, our remaining task is to show that any 5-chordal graph $G$ with $\delta^{*}(G)>\frac{1}{2}$ must contain one of $C_{4}, H_{1}, H_{2}, H_{3}$ and $H_{4}$ as an isometric subgraph. In view of Theorem 3 and Eqs. (2) and (4), we need only consider the case that $G$ contains a slingshot from $x$ to $y$, say $x, y, u, v$. We assume that this is the shortest slingshot in $G$ and base the subsequent argument on the notation as well as the claims given in Lemma 7.

Since $G$ is 5 -chordal and the cycle $C$ can have at most one chord (by Lemma 7), we know that the length $\ell$ of the slingshot is at most 4 . When $\ell=2$, the cycle $C$ is an induced $C_{4}$ of $G$, and hence by Lemma 6 (i), an isometric $C_{4}$. When $\ell=3$ or 4 , considering that $G$ is 5 -chordal, the cycle $C$ must have exactly one chord which connects $u_{2}$ and $v_{2}$. For the case of $\ell=3$, it follows from Lemma 6 (ii) that the subgraph induced by $P_{u} \cup P_{v}$ is an isometric $H_{3}$. As with the case of $\ell=4$, we first apply Lemma 7 (i) to get that 


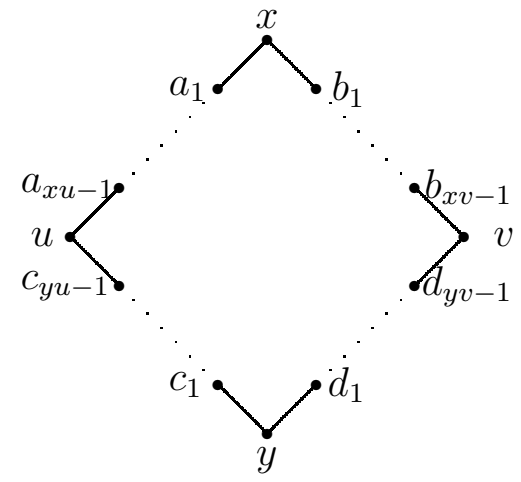

Figure 3: The geodesic quadrangle $\mathcal{Q}(x, u, y, v)$.

$u_{1} v_{3}=u_{3} v_{1}=3$ and then conclude from Lemma 6 (iii) that the subgraph induced by $P_{u} \cup P_{v}$ is an isometric $H_{4}$, completing the proof.

\section{Proof of Theorem 2}

We break the proof into several steps and so we will go through several lemmas and assumptions before we arrive at the final proof.

Let $G$ be a graph. When studying $\delta_{G}(x, y, u, v)$ for some vertices $x, y, u, v$ of $G$, it is natural to look at a geodesic quadrangle $\mathcal{Q}(x, u, y, v)$ with corners $x, u, y$ and $v$, which is just the subgraph of $G$ induced by the union of all those vertices on four geodesics connecting $x$ and $u, u$ and $y, y$ and $v$, and $v$ and $x$, respectively. Let us fix some notation to be used later.

Assumption I: Let us assume that $x, u, y, v$ are four different vertices of a graph $G$ and the four geodesics corresponding to the geodesic quadrangle $\mathcal{Q}(x, u, y, v)$ are

$$
\left\{\begin{array}{l}
P_{a}: x=a_{0}, a_{1}, \ldots, a_{x u}=u \\
P_{b}: x=b_{0}, b_{1}, \ldots, b_{x v}=v \\
P_{c}: y=c_{0}, c_{1}, \ldots, c_{y u}=u \\
P_{d}: y=d_{0}, d_{1}, \ldots, d_{y v}=v
\end{array}\right.
$$

We call $P_{a}, P_{b}, P_{c}$ and $P_{d}$ the four sides of $\mathcal{Q}(x, u, y, v)$ and often just think of them as vertex subsets of $V(G)$ rather than as vertex sequences. Let us say that $P_{a}$ and $P_{b}$ are adjacent to each other and refer to $x$ as their common peak; similar concepts are used in an obvious way. 


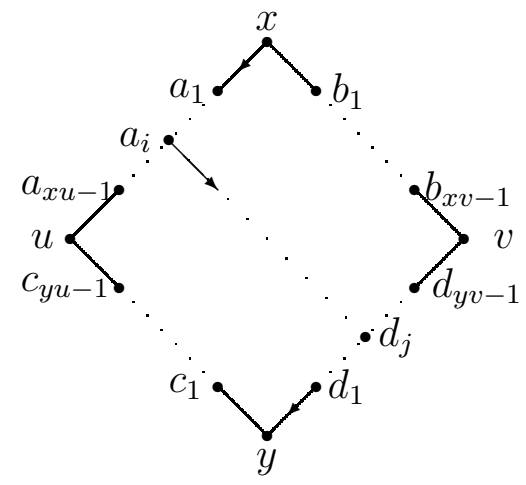

Figure 4: $x y \leq i+a_{i} d_{j}+j$.

Lemma 8 Let $G$ be a graph and let $\mathcal{Q}(x, u, y, v)$ be one of its geodesic quadrangles for which Assumption I holds. If

$$
2 \delta_{G}(x, y, u, v)=(x y+u v)-\max (x u+y v, x v+y u)
$$

then $\delta_{G}(x, y, u, v) \leq \min \left(d\left(P_{a}, P_{d}\right), d\left(P_{b}, P_{c}\right)\right)$.

Proof: Without loss of generality, we assume that there exist $i$ and $j$ such that

$$
a_{i} d_{j}=\min \left(d\left(P_{a}, P_{d}\right), d\left(P_{b}, P_{c}\right)\right) .
$$

It is clear that

$$
x y \leq x a_{i}+a_{i} d_{j}+d_{j} y=i+a_{i} d_{j}+j ;
$$

see Fig. 4. Analogously, we have

$$
u v \leq u a_{i}+a_{i} d_{j}+d_{j} v=(x u-i)+a_{i} d_{j}+(y v-j) .
$$

Henceforth, we arrive at the following:

$$
\begin{aligned}
2 \delta(x, y, u, v)= & (x y+u v)-\max (x u+y v, x v+y u) \quad(\text { By Eq. (5)) } \\
\leq & (x y+u v)-(x u+y v) \\
\leq & \left(i+a_{i} d_{j}+j\right)+\left((x u-i)+a_{i} d_{j}+(y v-j)\right) \\
& -(x u+y v) \quad(\text { By Eqs. (7) and }(8)) \\
= & 2 a_{i} d_{j} .
\end{aligned}
$$

Combining this with Eq. (6), we finish the proof of the lemma. 


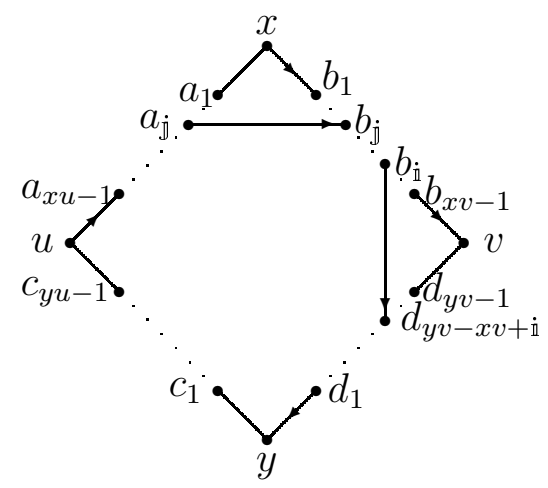

Figure 5: $x y \leq i+b_{i} d_{y v-x v+i}+(y v-(x v-i)), u v \leq(x u-j)+a_{j} b_{j}+(x v-j)$.

Lemma 9 Let $G$ be a graph and we will adopt Assumption I. We choose $\dot{\mathrm{i}}$ to be the minimum number such that $b_{\mathrm{i}} d_{y v-x v+\mathrm{i}} \leq 1, \mathfrak{j}$ the maximum number such that $a_{\mathfrak{j}} b_{\mathfrak{j}} \leq 1$, m the minimum number such that $a_{\mathrm{m}} c_{y u-x u+\mathrm{m}} \leq 1$, and $\mathrm{m}$ the maximum number such that $c_{\mathrm{m}} d_{\mathrm{m}} \leq 1$. Put

$$
\left\{\begin{array}{l}
\pi(a)=\mathrm{m}-\dot{j}+\frac{a_{\mathrm{j}} b_{\mathrm{j}}+a_{\mathrm{m}} c_{y u-x u+\mathrm{m}}}{2} \\
\pi(b)=\dot{\mathrm{i}}-\dot{\mathrm{j}}+\frac{a_{\mathrm{j}} b_{\mathrm{j}}+b_{\mathrm{i}} d_{y v-x v+\mathrm{i}}}{2} \\
\pi(c)=(y u-x u+\mathrm{m})-\mathrm{m}+\frac{a_{\mathrm{m}} c_{y u-x u+\mathrm{m}}+c_{\mathrm{n}} d_{\mathrm{m}}}{2} \\
\pi(d)=(y v-x v+\dot{\mathrm{i}})-\mathrm{m}+\frac{b_{\mathrm{i}} d_{y v-x v+\mathrm{i}+c_{\mathrm{n}} d_{\mathrm{m}}}}{2} .
\end{array}\right.
$$

If Eq. (5) is valid, then $\delta_{G}(x, y, u, v) \leq \min (\pi(a), \pi(b), \pi(c), \pi(d))$.

Proof: By symmetry, we only need to establish the inequality $\delta_{G}(x, y, u, v) \leq \pi(b)$. The crucial observation is as shown in Fig. 5, that is,

$$
\left\{\begin{array}{l}
x y \leq x b_{\dot{\mathbb{1}}}+b_{\dot{\mathrm{i}}} d_{y v-x v+\dot{\mathbb{i}}}+d_{y v-x v+\dot{\mathrm{i}}} y=\dot{\mathrm{i}}+b_{\dot{\mathrm{i}}} d_{y v-x v+\dot{\mathrm{i}}}+(y v-(x v-\dot{\mathrm{i}})) \\
u v \leq u a_{\mathfrak{j}}+a_{\dot{j}} b_{\dot{\mathfrak{j}}}+b_{\mathfrak{j}} v=(x u-\dot{\mathfrak{j}})+a_{\mathfrak{j}} b_{\mathfrak{j}}+(x v-\dot{\mathfrak{j}}) .
\end{array}\right.
$$

Accordingly, we have

$$
\begin{aligned}
2 \delta_{G}(x, y, u, v)= & (x y+u v)-\max (x u+y v, x v+y u) \quad(\text { By Eq. (5)) } \\
\leq & (x y+u v)-(x u+y v) \\
\leq & \left(\dot{\mathrm{i}}+b_{\dot{\mathrm{i}}} d_{y v-x v+\dot{\mathrm{i}}}+(y v-(x v-\dot{\mathrm{i}}))\right)+((x u-\dot{\mathfrak{j}}) \\
& \left.+a_{\dot{j}} b_{\dot{j}}+(x v-\dot{\mathfrak{j}})\right)-(x u+y v) \quad(\text { By Eq. }(10)) \\
= & 2 \pi(b),
\end{aligned}
$$

which is exactly what we want.

Brinkmann, Koolen and Moulton [14] introduce an extremality argument to deduce upper bounds of hyperbolicity of graphs. We follow their approach to make the following standing assumption in the main steps leading towards Theorem 2. 
Assumption II: We assume $x, y, u, v$ are four different vertices of $G$ such that the sum $x y+u v$ is minimal subject to the condition

$$
x y+u v=\max (x u+y v, x v+y u)+2 \delta^{*}(G) .
$$

The following key lemma of Brinkmann, Koolen and Moulton is found as a piece of their long proof of Theorem 1. We include a complete proof below, which is basically the one presented in [14], hoping to convince the readers that this lemma does hold in our more general setting.

Lemma 10 [14, p. 67, Claim 1] [61, p. 690, Claim 1] Let $G$ be any graph and u,v, x,y $\in$ $V(G)$. Under the Assumptions I and II, we have $a_{1} v \geq x v, a_{x u-1} y \geq u y, b_{1} u \geq x u$, $b_{x v-1} y \geq v y, c_{1} v \geq y v, c_{y u-1} x \geq u x, d_{1} u \geq y u, d_{y v-1} x \geq v x$.

Proof: By symmetry, we only need to show that $a_{1} v \geq x v$. If $a_{1} v<x v$, then, as a result of $a_{1} v \geq x v-x a_{1}=x v-1$, we have

$$
a_{1} v=x v-1 .
$$

Notice the obvious fact that

$$
a_{1} u=x u-1 .
$$

We then come to the following:

$$
\begin{aligned}
a_{1} y+u v & \geq\left(x y-x a_{1}\right)+u v \\
& =(x y-1)+u v \\
& =(x y+u v)-1 \\
& =\max (x u+y v-1, x v+y u-1)+2 \delta^{*}(G) \quad \text { (By Eq. (11)) } \\
& =\max \left(a_{1} u+y v, a_{1} v+y u\right)+2 \delta^{*}(G) . \quad \text { (By Eqs. (12) and (13)) }
\end{aligned}
$$

According to the definition of $\delta^{*}(G)$, we read from Eq. (14) that $a_{1} y+u v=\max \left(a_{1} u+\right.$ $\left.y v, a_{1} v+y u\right)+2 \delta^{*}(G)$ and hence that $a_{1} y+u v=x y+u v-1$. This contrasts with the minimality of the sum $x y+u v$ (Assumption II), completing the proof.

With the help of the previous lemma, we can derive the next one in a way similar to that of Lemma 7 (i).

Lemma 11 Suppose that Assumptions I and II are met. (i) Any two adjacent sides of $\mathcal{Q}(x, u, y, v)$ only intersect at their common peak. (ii) Let $w$ be the common peak of two adjacent sides $P$ and $P^{\prime}$ of $\mathcal{Q}(x, u, y, v)$. If it holds $\alpha \alpha^{\prime}=1$ for some $\alpha \in P \backslash\{w\}$ and $\alpha^{\prime} \in P^{\prime} \backslash\{w\}$, then $\alpha w=\alpha^{\prime} w$.

Proof: (i) By symmetry, it suffices to prove that $a_{p} \neq b_{q}$ for any $p \geq q>0$. Suppose otherwise, it then follows that $b_{1}, b_{2}, \ldots, b_{q}=a_{p}, a_{p+1}, \ldots, a_{x u}=u$ is a path connecting $b_{1}$ and $u$ and so $b_{1} u<x u$, violating Lemma 10. (ii) It is no loss to merely prove that if $i, j>0$ and $a_{i} b_{j}=1$ then $i=j$. In the case of $i>j, b_{1}, b_{2}, \ldots, b_{j}, a_{i}, a_{i+1}, \ldots, a_{x u}=u$ is a path connecting $b_{1}$ and $u$ of length smaller than $x u$, contrary to Lemma 10. Similarly, $i<j$ is impossible as well. 


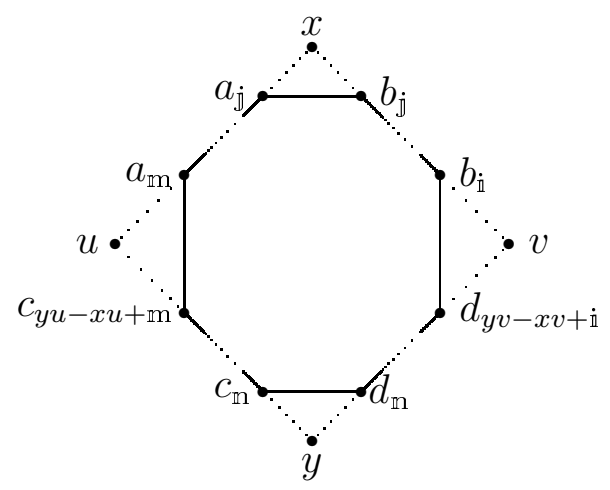

Figure 6: A chordless cycle in $\mathcal{Q}(x, u, y, v)$.

Lemma 12 Let $G$ be a $k$-chordal graph for some $k \geq 4$ and let $\mathcal{Q}(x, u, y, v)$ be a geodesic quadrangle for which Assumptions I and II hold. Let $\pi(a), \pi(b), \pi(c)$ and $\pi(d)$ be as defined in Eq. (9). Then we have

$$
\min (\pi(a), \pi(b), \pi(c), \pi(d)) \leq \frac{\left\lfloor\frac{k}{2}\right\rfloor}{2} .
$$

provided

$$
\min \left(d\left(P_{a}, P_{d}\right), d\left(P_{b}, P_{c}\right)\right)>1 .
$$

Proof: Suppose, for a contradiction, that the inequality (15) does not hold. In this event, as $\frac{\left\lfloor\frac{k}{2}\right\rfloor}{2} \geq 1$, we know that $\min (\mathrm{m}-\dot{j}, \dot{\mathrm{i}}-\dot{\mathfrak{j}},(y u-x u+\mathrm{m})-\mathrm{m},(y v-x v+\dot{\mathrm{i}})-\mathrm{m}) \geq$ $\min (\pi(a), \pi(b), \pi(c), \pi(d))>\frac{\left\lfloor\frac{k}{2}\right\rfloor}{2} \geq 1$. By virtue of Lemma 11 (i) and Eq. (16), this implies that

$$
C=\left[a_{\mathfrak{j}} b_{\mathfrak{j}} b_{\mathrm{j}+1} \cdots b_{\mathrm{i}} d_{y v-x v+\mathrm{i}} \cdots d_{\mathrm{m}-1} d_{\mathrm{m}} c_{\mathrm{m}} c_{\mathrm{m}+1} \cdots c_{y u-x u+\mathrm{m}} a_{\mathrm{m}} a_{\mathrm{m}-1} \cdots a_{\mathfrak{j}+1}\right]
$$

is a cycle, where the redundant $a_{j}$ should be deleted from the above notation when $a_{\mathfrak{j}}=b_{\mathrm{j}}=x$, the redundant $b_{\mathrm{i}}$ should be deleted from the above notation when $b_{\mathrm{i}}=$ $d_{y v-x v+\mathrm{i}}=v$, etc.; see Fig. 6. Moreover, by Lemma 11 (ii), Eq. (16) and the choice of $\dot{\mathrm{i}}, \dot{\mathrm{j}}, \mathrm{m}, \mathrm{m}$, we know that $C$ is even a chordless cycle. But the length of $C$ is just $\pi(a)+\pi(b)+\pi(c)+\pi(d)$, which, as the assumption is that (15) is violated, is no smaller than $4\left(\frac{1}{2}+\frac{\left\lfloor\frac{k}{2}\right\rfloor}{2}\right)$ and hence is at least $k+1$. This contradicts the assumption that $G$ is $k$-chordal, finishing the proof.

Proof of Theorem 2: Using typical compactness argument, it suffices to prove that every connected finite induced subgraph of a $k$-chordal graph $G$ is $\frac{\left\lfloor\frac{k}{2}\right\rfloor}{2}$-hyperbolic. Therefore, we can assume that $G$ is itself finite. If $\delta^{*}(G)=0$, then we are already finished. 


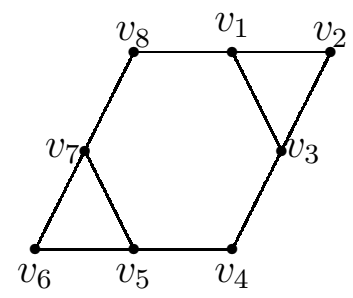

Figure 7: The outerplanar graph $F_{2}$ has chordality 6 , hyperbolicity $\frac{3}{2}$, and tree-length 2 .

Otherwise, there surely exists a geodesic quadrangle $\mathcal{Q}(x, u, y, v)$ in $G$ fulfilling Assumptions I and II. When $\min \left(d\left(P_{a}, P_{d}\right), d\left(P_{b}, P_{c}\right)\right) \leq 1$, the result is direct from Lemma 8 and the fact that $1 \leq \frac{\left\lfloor\frac{k}{2}\right\rfloor}{2}$; when $\min \left(d\left(P_{a}, P_{d}\right), d\left(P_{b}, P_{c}\right)\right)>1$, an application of Lemma 9 and Lemma 12 yields the required inequality.

\section{Tightness of Theorem 2}

We begin with an example from Brinkmann, Koolen and Moulton.

Example 13 [61, p. 683] For any $n \geq 3$, the chordality of $C_{n}$ is $n$ while the hyperbolicity of $C_{n}$ is $\left\lfloor\frac{n}{4}\right\rfloor-\frac{1}{2}$ for $n \equiv 1(\bmod 4)$ and $\left\lfloor\frac{n}{4}\right\rfloor$ else.

Example 14 For any $t \geq 2$ we set $F_{t}$ to be the outerplanar graph obtained from the $4 t$-cycle $\left[v_{1} v_{2} \cdots v_{4 t}\right]$ by adding the two edges $\left\{v_{1}, v_{3}\right\}$ and $\left\{v_{2 t+1}, v_{2 t+3}\right\}$; see Fig. 7 for an illustartion of $F_{2}$. Clearly, $\delta\left(v_{2}, v_{t+2}, v_{2 t+2}, v_{3 t+2}\right)=t-\frac{1}{2}$. Furthermore, we can check that $\mathbb{1}_{\mathbb{C}}\left(F_{t}\right)=4 t-2$ and $\delta^{*}\left(F_{t}\right)=t-\frac{1}{2}=\delta\left(v_{2}, v_{t+2}, v_{2 t+2}, v_{3 t+2}\right)=\frac{\mathbb{1}_{\mathbb{c}}\left(F_{t}\right)}{4}$.

It is clear that if the bound claimed by Theorem 2 is tight for $k=4 t(k=4 t-2)$ then it is tight for $k=4 t+1(k=4 t-1)$. Consequently, Examples 13 and 14 indeed mean that the bound reported in Theorem 2 is tight for every $k \geq 4$.

For any graph $G$ and any positive number $t$, we put $S^{t}(G)$ to be a subdivision graph of $G$, which is obtained from $G$ by replacing each edge $\{u, v\}$ of $G$ by a path $u, n_{u, v}^{1}, \ldots, n_{u, v}^{t-1}, v$ of length $t$ connecting $u$ and $v$ through a sequence of new vertices $n_{u, v}^{1}, \ldots, n_{u, v}^{t-1}$ (we surly require that $n_{v, u}^{q}=n_{u, v}^{t-q}$ ). For any four vertices $x, y, u, v \in V(G)$, we obviously have $\delta_{S^{t}(G)}(x, y, u, v)=t \delta_{G}(x, y, u, v)$ and so $\delta^{*}\left(S^{t}(G)\right) \geq t \delta^{*}(G)$. Instead of the trivial fact $\mathbb{l}_{\mathbb{C}}\left(S^{t}(G)\right) \geq t \mathbb{1}_{\mathbb{C}}(G)$, if the good shape of $G$ permits us to deduce a good upper bound of $\mathbb{l} \mathbb{C}\left(S^{t}(G)\right)$ in terms of $\mathbb{1} \mathbb{C}(G)$, we will see that $\delta^{*}\left(S^{t}(G)\right)$ is high relative to $\mathbb{l} \mathbb{C}\left(S^{t}(G)\right)$ provided so is $G$. Recall that the cycles whose lengths are divisible by 4 as discussed in Example 13 are used to demonstrate the tightness of the bound given in Theorem 2; also observe that the graphs suggested by Example 14 is nothing but a slight "perturbation" of cycles of length divisible by 4 . Since $C_{4 t}=S^{t}\left(C_{4}\right)$, these examples can be said to be 


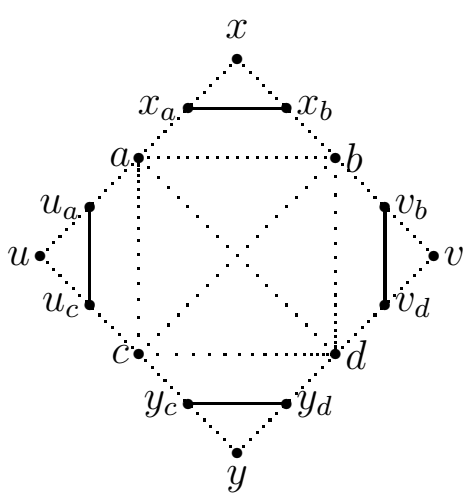

Figure 8: $\mathbb{G}_{4 t}^{q}$.

generated by the "seed" $C_{4}$. It might deserve to look for some other good "seeds" from which we can use the above subdivision operation or its variant to produce graphs $G$ satisfying Eq. (3).

The rest of this section aims to provide more constructions showing the tightness of Theorem 2. The next example is suggested by Gavoille, which uses $H_{2}$ as the "seed".

Example 15 Let $t, q$ be two positive integer with $q<t$ and let $H_{2}$ be the graph shown in the upper-right corner of Fig. 1. We construct a planar graph $\mathbb{G}_{4 t}^{q}$ from $S^{t}\left(H_{2}\right)$ as follows: let $u_{a}=n_{u, a}^{q}, u_{c}=n_{c, u}^{q-1}, y_{c}=n_{y, c}^{q}, y_{d}=n_{d, y}^{q-1}, v_{d}=n_{v, d}^{q}, v_{b}=n_{b, v}^{q-1}, x_{b}=n_{x, b}^{q}, x_{a}=$ $n_{a, x}^{q-1}$, and then add the new edges $\left\{u_{a}, u_{c}\right\},\left\{x_{a}, x_{b}\right\},\left\{y_{c}, y_{d}\right\},\left\{v_{b}, v_{d}\right\}$ to $S^{t}\left(H_{2}\right)$; see Fig. 8. It can be checked that $C=\left[u_{a} \cdots a \cdots x_{a} x_{b} \cdots b \cdots v_{b} v_{d} \cdots d \cdots y_{d} y_{c} \cdots c \cdots u_{c}\right]$ is an isometric $4 t$-cycle of $\mathbb{G}_{4 t}^{q}$ and that $\mathbb{1} \mathbb{C}\left(\mathbb{G}_{4 t}^{q}\right)=4 t$. It is also easy to see that $\delta_{\mathbb{G}_{4 t}^{q}}(u, y, v, x)=t$ and thus Theorem 2 tells us that $\delta^{*}\left(\mathbb{G}_{4 t}^{q}\right)=t$.

Motivated by the above construction of Gavoille, we discover the next graph family whose chordality parameters are 1 modulo 4 .

Example 16 By deleting the edge $\left\{y_{c}, n_{d, y}^{q-1}\right\}$ and adding a new edge $\left\{y_{c}, n_{d, y}^{q}\right\}$, we obtain from $\mathbb{G}_{4 t}^{q}$ a graph $\mathbb{G}_{4 t+1}^{q}$. Using similar analysis like Example 15 , we find that $\mathbb{1} \mathbb{C}\left(\mathbb{G}_{4 t}^{q}\right)=$ $4 t+1$ and $\delta^{*}\left(\mathbb{G}_{4 t+1}^{q}\right)=t=\frac{\left\lfloor\frac{4 t+1}{2}\right\rfloor}{2}$.

To get extremal graphs whose chordality parameters are 2 or 3 modulo 4 , we can use $F_{2}$ (see Fig. 7) as the "seed".

Example 17 Let $t>q$ be two positive integers. We construct an outerplanar graph $\mathbb{G}_{6(2 t+1)}^{q}$ by adding two new edges $\left\{v_{21}, v_{23}\right\}$ and $\left\{v_{65}, v_{67}\right\}$ to the graph $S^{2 t+1}\left(F_{2}\right)$ where $v_{21}=n_{v_{2}, v_{1}}^{q}, v_{23}=n_{v_{3}, v_{2}}^{q-1}, v_{65}=n_{v_{6}, v_{5}}^{q}, v_{67}=n_{v_{7}, v_{6}}^{q-1}$; see Fig. 9 for an illustration. It is not hard to check that $\mathbb{1}_{\mathbb{C}}\left(\mathbb{G}_{6(2 t+1)}^{q}\right)=6(2 t+1)$ and $\delta^{*}\left(\mathbb{G}_{6(2 t+1)}^{q}\right)=3 t+\frac{3}{2}$. Moreover, if we replace the edge $\left\{v_{21}, v_{23}\right\}$ by the edge $\left\{v_{21}, n_{v_{3}, v_{2}}^{q}\right\}$, then we obtain from $\mathbb{G}_{6(2 t+1)}^{q}$ another outerplanar graph $\mathbb{G}_{6(2 t+1)+1}^{q}$ for which we have $\mathbb{1} \mathbb{C}\left(\mathbb{G}_{6(2 t+1)+1}^{q}\right)=6(2 t+1)+1$ and $\delta^{*}\left(\mathbb{G}_{6(2 t+1)+1}^{q}\right)=3 t+\frac{3}{2}$. 


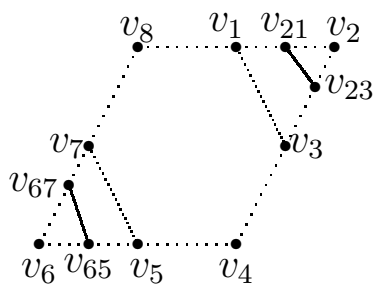

Figure 9: $\mathbb{G}_{6(2 t+1)}^{q}$.

\section{Graph classes with low chordality}

An asteroidal triple $(A T)$ of a graph $G$ is a a set of three vertices of $G$ such that for any pair of them there is a path connecting the two vertices and having a distance at least two to the remaining vertex. A graph is $A T$-free if no three vertices form an $A T$ [12, p. 114]. Obviously, all $A T$-free graphs are 5-chordal. A graph is an interval graph exactly when it is both chordal and $A T$-free [12, Theorem 7.2.6]. AT-free graphs also include cocomparability graphs [12, Theorem 7.2.7]; moreover, all bounded tolerance graphs are cocomparability graphs [46] [47, Theorem 2.8] and a graph is a permutation graph if and only if itself and its complement are cocomparability graphs [12, Theorem 4.7.1]. An important subclass of cocomparability graphs is the class of threshold graphs, which are those graphs without any induced subgraph isomorphic to the 4-cycle, the complement of the 4-cycle or the path of length 3 [47, p. 23].

A graph is weakly chordal [46, 51] when both itself and its complement are 4-chordal. Note that all tolerance graphs [47] are domination graphs [72] and all domination graphs are weakly chordal [25]. An odd chord of a cycle of even length is a chord connecting different vertices the distance between which in the cycle is odd. A graph is strongly chordal if it is chordal and if every even cycle of length at least 6 in this graph has an odd chord [46, p. 21]. A graph is distance-hereditary if each of its induced paths, and hence each of its connected induced subgraphs, is isometric [53]. We call a graph a cograph provided it does not contain any induced path of length 3 [12, Theorem 11.3.3]. It is easy to see that each cograph is distance-hereditary and all distance-hereditary graphs form a proper subclass of 4-chordal graphs. It is also known that cocomparability graphs are all 4-chordal [10, 43].

Corollary 18 Each weakly chordal graph is 1-hyperbolic and has hyperbolicty one if and only if it contains one of $\mathrm{C}_{4}, \mathrm{H}_{1}, \mathrm{H}_{2}$ as an isometric subgraph.

Proof: By definition, each weakly chordal graph is 4-chordal. It is also easy to check that that $C_{4}, H_{1}$ and $H_{2}$ are all weakly chordal. Hence, the result follows from Corollary 5 .

Corollary 19 All strongly chordal graphs are $\frac{1}{2}$-hyperbolic. 
Proof: Note that the even cycle $C=[x, a, u, c, y, d, v, b]$ in $H_{1}$ and $H_{2}$ does not have any odd chord and hence neither $H_{1}$ nor $H_{2}$ can appear as an induced subgraph of a strongly chordal graph. Since strongly chordal graphs must be chordal graphs, this result holds by Theorem 1.

Corollary 20 All threshold graphs are $\frac{1}{2}$-hyperbolic.

Proof: It is obvious that threshold graphs are chordal as they contain neither 4-cycle nor path of length 3 as induced subgraph. Since the subgraph induced by $x, u, b, c$ in either $H_{1}$ or $H_{2}$ is just the complement of $C_{4}$, the result follows from Theorem 1 and the definition of a threshold graph.

Corollary 21 Every AT-free graph is 1-hyperbolic and it has hyperbolicity one if and only if it contains $C_{4}$ as an isometric subgraph.

Proof: First observe that an $A T$-free graph must be 5-chordal. Further notice that the triple $u, y, v$ is an $A T$ in any of the graphs $H_{1}, H_{2}, H_{3}$, and $H_{4}$. Now, an application of Theorem 4 concludes the proof.

Corollary 22 A cocomparability graph is 1-hyperbolic and has hyperbolicity one if and only if it contains $C_{4}$ as an isometric subgraph.

Proof: We know that cocomparability graphs are $A T$-free and $C_{4}$ is a cocomparability graph. Thus the result comes directly from Corollary 21. The deduction of this result can also be made via Corollary 5 and the fact that cocomparability graphs are 4-chordal $[10,43]$.

Corollary 23 A permutation graph is 1-hyperbolic and has hyperbolicity one if and only if it contains $C_{4}$ as an isometric subgraph.

Proof: Every permutation graph is a cocomparability graph and $C_{4}$ is a permutation graph. So, the result follows from Corollary 22.

Corollary 24 [7, p. 16] A distance-hereditary graph is always 1-hyperbolic and is $\frac{1}{2}$ hyperbolic exactly when it is chordal, or equivalently, when it contains no induced 4-cycle.

Proof: It is easy to see that distance-hereditary graphs must be 4-chordal and can contain neither $H_{1}$ nor $H_{2}$ as an isometric subgraph. The result now follows from Corollary 5 . 
Corollary 25 A cograph is 1-hyperbolic and has hyperbolicity one if and only if it contains $C_{4}$ as an isometric subgraph.

Proof: We know that $C_{4}$ is a cograph and every cograph is ditance-hereditary. Applying Corollary 24 yields the required result.

Acknowledgements We thank Feodor Dragan and Cyril Gavoille for some useful communications when we are preparing this paper. Y. Wu thanks Michel Deza for inviting him to deliver a talk on this work in the Workshop on Metric Graph Theory, Kanazawa, Japan, November 11-13, 2009. One referee suggests us deduce Theorem 4 directly from Theorem 3, which enables us to replace our original self-contained proof of Theorem 4 by the current significantly shorter argument. This work is supported by Science and Technology Commission of Shanghai Municipality (No. 08QA14036 and No. 09XD1402500), Chinese Ministry of Education (No. 108056), and National Natural Science Foundation of China (No. 10871128).

\section{References}

[1] I. Abraham, M. Balakrishnan, F. Kuhn, D. Malkhi, V. Ramasubramanian, K. Talwar, Reconstructing approximate tree metrics, PODC 2007, 43-52.

[2] J.M. Alonso, T. Brady, D. Cooper, V. Ferlini, M. Lustig, M. Mihalik, M. Shapiro, H. Short, Notes on word hyperbolic groups, Group Theory from a Geometrical Viewpoint, ICTP Trieste 1990 (E. Ghys, A. Haefliger, A. Verjovsky, eds.), World Scientific, 1991, pp. 3-63. Available at: http://homeweb1.unifr.ch/ciobanul/pub/Teaching/ kggt/MSRInotes2004.pdf

[3] R.P. Anstee, M. Farber, On bridged graphs and cop-win graphs, Journal of Combinatorial Theory A 44 (1988), 22-28.

[4] S. Arnborg, J. Lagergren, D. Seese, Easy problems for tree-decomposable graphs, Journal of Algorithms 12 (1991), 308-340.

[5] P. Bahls, Asymptotic connectivity of infinite graphs, Discrete Mathematics 309 (2009), 2250-2259.

[6] H-J. Bandelt, V. Chepoi, 1-Hyperbolic graphs, SIAM Journal on Discrete Mathematics 16 (2003), 323-334.

[7] H-J. Bandelt, V. Chepoi, Metric graph theory and geometry: A survey, in: Surveys on Discrete and Computational Geometry: Twenty Years Later, J.E. Goodman, J. Pach, R. Pollack (eds.), AMS, pp. 49-86, 2008.

[8] H-J. Bandelt, H.M. Mulder, Distance-hereditary graphs, Journal of Combinatorial Theory B 41 (1986), 182-208.

[9] H.L. Bodlaender, J. Engelfriet, Domino treewidth, Journal of Algorithms 24 (1997), 94-123.

[10] H.L. Bodlaender, D.M. Thilikos, Treewidth for graphs with small chordality, Discrete Applied Mathematics 79 (1997), 45-61.

[11] B. Bowditch, Notes on Gromov's hyperbolicity criterion for path-metric spaces, in: Group Theory from a Geometrical Viewpoint, E. Ghys, A. Haefliger, A. Verjovsky (eds.), World Scientific, Singapore, pp. 64-167, 1991. 
[12] A. Brandstädt, V.B. Le, J.P. Spinrad, Graph Classes: A Survey, SIAM, 1999.

[13] M. Bridson, A. Haefliger, Metric Spaces of Non-Positive Curvature, Springer, Berlin, 1999.

[14] G. Brinkmann, J.H. Koolen, V. Moulton, On the hyperbolicity of chordal graphs, Annals of Combinatorics 5 (2001), 61-69.

[15] L.S. Chandran, V.V. Lozin, C.R. Subramanian, Graphs of low chordality, Discrete Mathematics $\&$ Theoretical Computer Science 7 (2005), 25-36.

[16] L.S. Chandran, L.S. Ram, On the number of minimum cuts in a graph, SIAM Journal on Discrete Mathematics 18 (2004), 177-194.

[17] M-S. Chang, H. Müller, On the tree-degree of graphs, Lecture Notes in Computer Science 2204 (2001), 44-54.

[18] B. Chen, S.-T. Yau, Y.-N. Yeh, Graph homotopy and Graham homotopy, Discrete Mathematics 241 (2001), 153-170.

[19] H. Chen, V. Dalmau, Beyond hypertree width: Decomposition methods without decompositions, Lecture Notes in Computer Science 3709 (2005), 167-181.

[20] V. Chepoi, F. Dragan, B. Estellon, M. Habib, Y. Vaxés, Notes on diameters, centers, and approximating trees of $\delta$-hyperbolic geodesic spaces and graphs, Electronic Notes in Discrete Mathematics 31 (2008), 231-234.

[21] V. Chepoi, F. Dragan, B. Estellon, M. Habib, Y. Vaxés, Diameters, centers, and approximating trees of $\delta$-hyperbolic geodesic spaces and graphs, Annual Symposium on Computational Geometry pp. 59-68, 2008.

[22] V. Chepoi, F.F. Dragan, B. Estellon, M. Habib, Y. Vaxés, Y. Xiang, Additive spanners and distance and routing labeling schemes for hyperbolic graphs, submitted. Available at: http://pageperso.lif . univ-mrs.fr/ victor . chepoi/HypRoutJournal . pdf

[23] V. Chepoi, B. Estellon, Packing and covering $\delta$-hyperbolic spaces by balls, Lecture Notes in Computer Science 4627 (2007), 59-73.

[24] D. Cohen, P. Jeavons, M. Gyssens, A unified theory of structural tractability for constraint satisfaction problems, Journal of Computer and System Sciences 74 (2008), 721-743.

[25] E. Dahlhaus, P.L. Hammer, F. Maffray, S. Olariu, On domination elimination orderings and domination graphs, Lecture Notes in Computer Science 903 (1994), 81-92.

[26] N.D. Dendris, L.M. Kirousis, D.M. Thilikos, Fugitive-search games on graphs and related parameters, Theoretical Computer Science 172 (1997), 233-254.

[27] M.M. Deza, E. Deza, Encyclopedia of Distances, Springer, 2009.

[28] R. Diestel, Graph Theory, Springer, 1997.

[29] M. Dinitz, Online, dynamic, and distributed embeddings of approximate ultrametrics, Lecture Notes in Computer Science 5218 (2008), 152-166.

[30] Y. Dourisboure, C. Gavoille, Tree-decompositions with bags of small diameter, Discrete Mathematics 307 (2007), 2008-2029.

[31] R.G. Downey, C. McCartin, Bounded persistence pathwidth, in: M. Atkinson and F. Dehne, editors, Eleventh Computing: The Australasian Theory Symposium (CATS2005), volume 41 of CRPIT, pages 51-56, Newcastle, Australia, 2005. ACS (Australian Computer Society).

THE ELECTRONiC Journal of COMBinatorics 18 (2011), \#P43 
[32] R.G. Downey, C. McCartin, Online promise problems with online width metrics, Journal of Computer and System Sciences 73 (2007), 57-72.

[33] F.F. Dragan, Y. Xiang, How to use spanning trees to navigate in graphs, Lecture Notes in Computer Science 5734 (2009), 282-294.

[34] A. Dress, Trees, tight extensions of metric spaces, and the cohomological dimension of certain groups: A note on combinatorial properties of metric spaces, Advances in Mathematics 53 (1984), 321-402.

[35] A. Dress, B. Holland, K.T. Huber, J.H. Koolen, V. Moulton, J. Weyer-Menkhoff, $\Delta$ additive and $\Delta$ ultra-additive maps, Gromov's trees, and the Farris transform, Discrete Applied Mathematics 146 (2005), 51-73.

[36] A. Dress, K.T. Huber, V. Moulton, Some uses of the Farris transform in mathematics and phylogenetics - A Review, Annals of Combinatorics 11 (2007), 1-37.

[37] A. Dress, V. Moulton, M. Steel, Trees, taxonomy, and strongly compatible multi-state characters, Advances in Applied Mathematics 19 (1997), 1-30.

[38] A. Dress, V. Moulton, W. Terhalle, T-theory: An overview, European Journal of Combinatorics 17 (1996), 161-175.

[39] R. Duke, Types of cycles in hypergraphs, Annals of Discrete Mathematics 27 (1985), 399418.

[40] R. Fagin, Degrees of acyclicity for hypergraphs and relational database schemes, Journal of the Association for Computing Machinery 30 (1983), 514-550.

[41] M. Farber, R.E. Jamison, On local convexities in graphs, Discrete Mathematics 66 (1987), 231-247.

[42] J.S. Farris, A.G. Kluge, M.J. Eckardt, A numerical approach to phylogenetic systematics, Systematic Zoology (continued by Syst. Biol.) 19 (1970), 172-189.

[43] T. Gallai, Transitiv orientierbare Graphen, Acta Mathematica Academiae Scientiarum Hungaricae (continued by Acta Math. Hungar.) 18 (1967), 25-66.

[44] C. Gavoille, O. Ly, Distance labeling in hyperbolic graphs, Lecture Notes in Computer Science 3827 (2005), 1071-1079.

[45] F. Gavril, Algorithms for maximum weight induced paths, Information Processing Letters 81 (2002), 203-208.

[46] M.C. Golumbic, C.L. Monma, W.T. Trotter, Tolerance graphs, Discrete Applied Mathematics 9 (1984), 157-170.

[47] M.C. Golumbic, A.N. Trenk, Tolerance Graphs, Cambridge University Press, Cambridge, 2004.

[48] G. Gottlob, N. Leone, F. Scarcello, Hypertree decompositions and tractable queries, Journal of Computer and System Sciences 64 (2002), 579-627.

[49] M. Gromov, Hyperbolic groups, in: Essays in Group Theory, S. Gersten (ed.), MSRI Series, 8, Springer-Verlag, pp. 75-263, 1987.

[50] A. Gupta, R. Krauthgamer, J.R. Lee, Bounded geometries, fractals, and low-distortion embeddings, 44th Annual IEEE Symposium on Foundations of Computer Science (FOCS'03), pp. 534-543, 2003. 
[51] R.B. Hayward, Weakly triangulated graphs, Journal of Combinatorial Theory B 39 (1985), 200-208.

[52] P. Hliněný, S-I. Oum, D. Seese, G. Gottlob, Width parameters beyond tree-width and their applications, The Computer Journal 51 (2008), 326-362.

[53] E. Howorka, On metric properties of certain clique graphs, Journal of Combinatorial Theory B 27 (1979), 67-74.

[54] P. Hunter, S. Kreutzer, Digraph measures: Kelly decompositions, games, and orderings, Theoretical Computer Science 399 (2008), 206-219.

[55] W. Imrich, On metric properties of tree-like spaces, Contribution to graph theory and its applications (Internat. Colloq. Oberhof, 1977) pp. 129-156, Tech. Hochschule Ilmenau, Ilmenau, 1977.

[56] E. Jonckheere, P. Lohsoonthorn, F. Bonahon, Scaled Gromov hyperbolic graphs, Journal of Graph Theory 57 (2008), 157-180.

[57] E. Jonckheere, M. Lou, J. Hespanha, P. Barooah, Effective resistance of Gromov-hyperbolic graphs: Application to asymptotic sensor network problems, 46th IEEE Conference on Decision and Control (2007), 1453-1458.

[58] L.M. Kirousis, D.M. Thilikos, The linkage of a graph, SIAM Journal on Computing 25 (1996), 626-647.

[59] V. Klee, What is the maximum length of a $d$-dimensional snake? The American Mathematical Monthly 77 (1970), 63-65.

[60] R. Kleinberg, Geographic routing using hyperbolic space, INFOCOM (2007), 1902-1909.

[61] J.H. Koolen, V. Moulton, Hyperbolic bridged graphs, European Journal of Combinatorics 23 (2002), 683-699.

[62] F.R. Kschischang, B.J. Frey, H-A. Loeliger, Factor graphs and the sum-product algorithm, IEEE Transactions on Information Theory 47 (2001), 498-519.

[63] M. Laurent, On the sparsity order of a graph and its deficiency in chordality, Combinatorica 21 (2001), 543-570.

[64] V.B. Le, J. Spinrad, Consequence of an algorithm for bridged graphs, Discrete Applied Mathematics 280 (2004), 271-274.

[65] T.T. Lee, The Euler formula of cyclomatic numbers of hypergraphs, Southeast Asian Bulletin of Mathematics 21 (1997), 113-137.

[66] D.R. Lick, A.T. White, k-Degenerate graphs, Canadian Journal of Mathematics 22 (1970), 1082-1096.

[67] T.A. McKee, E.R. Scheinerman, On the chordality of a graph, Journal of Graph Theory 17 (1993), 221-232.

[68] V. Moulton, M. Steel, Retractions of finite distance functions onto tree metrics, Discrete Applied Mathematics 91 (1999), 215-233.

[69] F.S. Roberts, On the boxicity and cubicity of a graph, in: Recent Progresses in Combinatorics, Academic Press, New York, 1969, pp. 301-310.

[70] N. Robertson, P.D. Seymour, Graph minors. III. Planar tree-width, Journal of Combinatorial Theory B 36 (1984), 49-63. 
[71] N. Robertson, P.D. Seymour, Graph minors. II. Algorithmic aspects of tree-width, Journal of Algorithms 7 (1986), 309-322.

[72] I. Rusu, J. Spinrad, Domination graphs: Examples and counterexamples, Discrete Applied Mathematics 110 (2001), 289-300.

[73] Y. Shavitt, T. Tankel, Hyperbolic embedding of internet graph for distance estimation and overlay construction, IEEE/ACM Transactions on Networking 16 (2008), 25-36.

[74] V.P. Soltan, V.D. Chepoi, Conditions for invariance of set diameters under $d$-convexification in a graph, Cybernetics 19 (1983), 750-756.

[75] M. Thorup, All structured programs have small tree-width and good register allocation, Information and Computation 142 (1998), 159-181.

[76] R. Uehara, Tractable and intractable problems on generalized chordal graphs, IEICE Technical Report, COMP98-83, pages 1-8, 1999. Available at: http://www.jaist.ac.jp/ $\sim$ uehara/pub/tech.html

[77] K. Umezawa, K. Yamazaki, Tree-length equals branch-length, Discrete Mathematics 309 (2009), 4656-4660.

[78] J. Väisälä, Gromov hyperbolic spaces, Expositiones Mathematicae 23 (2005), 187-231.

[79] D.R. Wood, On tree-partition-width, European Journal of Combinatorics 30 (2009), 12451253.

[80] A. Yamaguchi, K.F. Aoki, H. Mamitsuka, Graph complexity of chemical compounds in biological pathways, Genome Informatics 14 (2003), 376-377. 